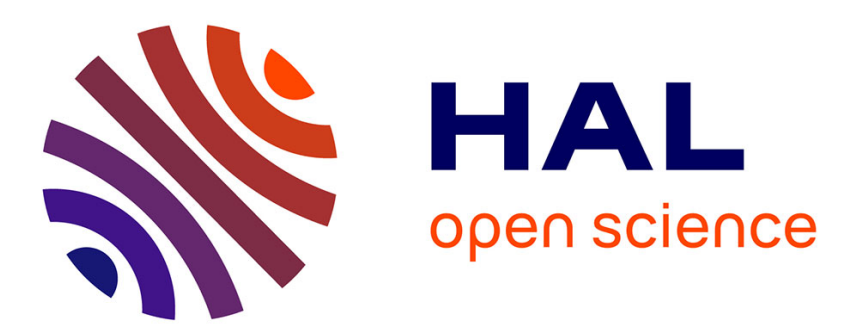

\title{
Identifying local characteristic lengths governing sound wave properties in solid foams
}

\author{
M. T. Hoang, Camille Perrot
}

\section{To cite this version:}

M. T. Hoang, Camille Perrot. Identifying local characteristic lengths governing sound wave properties in solid foams. Journal of Applied Physics, 2013, 113 (8), pp.084905-7. 10.1063/1.4793492 . hal00799631

\section{HAL Id: hal-00799631 \\ https://hal.science/hal-00799631}

Submitted on 12 Mar 2013

HAL is a multi-disciplinary open access archive for the deposit and dissemination of scientific research documents, whether they are published or not. The documents may come from teaching and research institutions in France or abroad, or from public or private research centers.
L'archive ouverte pluridisciplinaire HAL, est destinée au dépôt et à la diffusion de documents scientifiques de niveau recherche, publiés ou non, émanant des établissements d'enseignement et de recherche français ou étrangers, des laboratoires publics ou privés. 


\section{AIP Applied Physics}

\section{Identifying local characteristic lengths governing sound wave properties in solid foams}

Minh Tan Hoang and Camille Perrot

Citation: J. Appl. Phys. 113, 084905 (2013); doi: 10.1063/1.4793492

View online: http://dx.doi.org/10.1063/1.4793492

View Table of Contents: http://jap.aip.org/resource/1/JAPIAU/v113/i8

Published by the American Institute of Physics.

\section{Related Articles}

A stable method to model the acoustic response of multilayered structures

J. Appl. Phys. 113, 083506 (2013)

Method of extreme surfaces for optimizing geometry of acousto-optic interactions in crystalline materials:

Example of LiNbO3 crystals

J. Appl. Phys. 113, 083103 (2013)

Photothermal model fitting in the complex plane for thermal properties determination in solids

Rev. Sci. Instrum. 84, 024903 (2013)

Transient lattice distortion induced by ultrashort heat pulse propagation through thin film metal/metal interface Appl. Phys. Lett. 102, 051915 (2013)

Lamb wave band gaps in a double-sided phononic plate

J. Appl. Phys. 113, 053509 (2013)

\section{Additional information on J. Appl. Phys.}

Journal Homepage: http://jap.aip.org/

Journal Information: http://jap.aip.org/about/about_the_journal

Top downloads: http://jap.aip.org/features/most_downloaded

Information for Authors: http://jap.aip.org/authors

\section{ADVERTISEMENT}

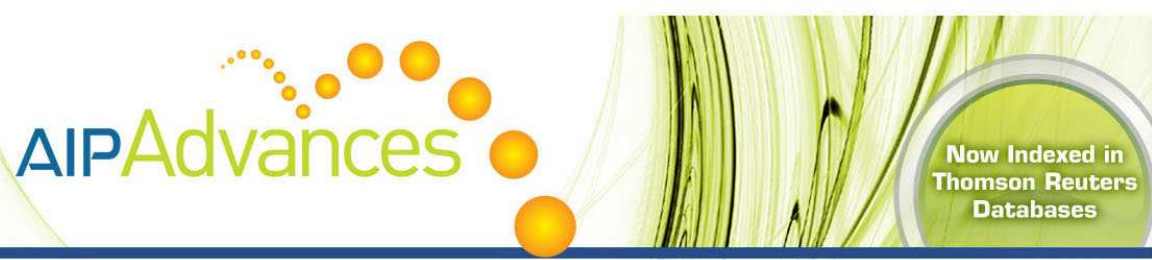

\section{Explore AIP's open access journal: •Rapid publication \\ - Article-level metrics \\ - Post-publication rating and commenting}




\title{
Identifying local characteristic lengths governing sound wave properties in solid foams
}

\author{
Minh Tan Hoang ${ }^{1,2}$ and Camille Perrot ${ }^{1, a)}$ \\ ${ }^{1}$ Université Paris-Est, Laboratoire Modélisation et Simulation Multi Echelle, MSME UMR 8208 CNRS, \\ 5 bd Descartes, 77454 Marne-la-Vallée, France \\ ${ }^{2}$ Faurecia Interior Systems, Acoustic TechCenter, Z.I. François Sommer BP 13, 08210 Mouzon, France
}

(Received 22 August 2012; accepted 11 February 2013; published online 26 February 2013)

\begin{abstract}
Identifying microscopic geometric properties and fluid flow through opened-cell and partially closed-cell solid structures is a challenge for material science, in particular, for the design of porous media used as sound absorbers in building and transportation industries. We revisit recent literature data to identify the local characteristic lengths dominating the transport properties and sound absorbing behavior of polyurethane foam samples by performing numerical homogenization simulations. To determine the characteristic sizes of the model, we need porosity and permeability measurements in conjunction with ligament lengths estimates from available scanning electron microscope images. We demonstrate that this description of the porous material, consistent with the critical path picture following from the percolation arguments, is widely applicable. This is an important step towards tuning sound proofing properties of complex materials. (C) 2013 American Institute of Physics. [http://dx.doi.org/10.1063/1.4793492]
\end{abstract}

\section{INTRODUCTION}

Some important characteristics of a sound absorbing material are the transport properties, from which an approximate but robust description of its long wavelengths behavior derives. ${ }^{1-4}$ Transport properties include the rate of either diffusive, electrical, or fluid movement through fluid-filled pore space. But, a more detailed understanding comes from identifying other microstructural parameters. For disordered rigid-framed solid foams in which a sound wave is propagating and dissipated by visco-inertial and thermal interactions, transport properties are often very sensitive to the local variability of the geometry, their real microstructure. Thus, a systematic identification of local characteristic lengths has not yet been performed in polyurethane foams. In this paper, we present precise estimates of microscopic geometric quantities of a three-dimensional periodic unit-cell (3D PUC) with representations of pore and throat sizes that explicitly show the microphysical basis behind transport phenomena.

In classical semi-phenomenological acoustics, no clear relationships occur with the microgeometry itself. The situation is quite different in numerical homogenization. Indeed, Auriault, ${ }^{5}$ and others (see references therein) showed that even a disordered porous medium can undergo a homogenization to a quite simple form, a periodic structure, provided that the situation obeys scale separation (any feature of the local geometry is much smaller compared to the incident wavelength). The application of this theory to real rigid porous media in the long wavelengths' regime captures the essential of the physics at both the micro and macro scales, and the way to compute explicitly the coefficients involved at the upper scale level. ${ }^{6,7}$

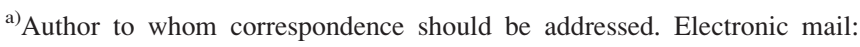
camille.perrot@univ-paris-est.fr.
}

Porous media that obey scale separations, such as disordered solid foam samples, undergo a transition between viscous (isothermal) and inertial (adiabatic) regimes by forming the three-dimensional periodic unit cells that act as the overall porous material. For this to happen, local characteristic lengths identification is necessary. In the simplest cases, the transport parameters are determined experimentally. ${ }^{8}$ This characterization may be correlated to the closed pore content of polyurethane foam samples (the ratio of the number of closed windows to the total number of windows visible on micrographs). ${ }^{9}$ The resulting semi-empirical relationships are typically applicable to a wide range of real foam samples. However, the micro-macro relations emerging from these correlations are only directly applicable to the actual fabrication processes. The physical insight in this numerical homogenization scheme is much larger than experimental characterization and semi-empirical correlations by allowing identification of local geometry features which have a significant impact at the upper scale.

The application of the numerical homogenization has proven to be widely applicable to synthesized periodic porous materials. ${ }^{10}$ Beyond conventional characterization techniques, ${ }^{11}$ it describes the complex interplay of the fluid flow within the porous media, in the frequency-dependent response, or in asymptotic behaviors at low and high frequencies. There is an important class of synthetic porous materials that do not follow the periodic structures paradigm. These materials seem too disordered to be described by a periodic unit cell that starts from a regular structure. However, a completely new way to interpret transports within disordered pore spaces has emerged-critical path ideas, whose conceptual strength can be used to identify characteristic lengths of the pore space. ${ }^{12,13}$ To determine the characteristic lengths of real solid foam samples, one model is used here to cope with different experimental 
studies in an iterative loop of experimentation and computations without any adjustable constant. ${ }^{14,15}$ Hoang and Perrot describe this class of model, using the example of three real foam samples which may contain solid films. ${ }^{15}$

\section{MATERIALS AND METHOD}

The general strategy to determine the three-dimensional periodic unit cell representative of a given real foam sample is to measure the porosity $\phi$ and permeability $k_{0}$, in such a way that a Kelvin cell without solid films corresponding to the measured experimental data might be found. Equivalently, by causing the closure rate of membranes $\delta / \delta_{\max }$ to grow, a relative measure of the membranes' size when compared to the size of the smallest windows, ${ }^{15}$ one can determine the new size of the cell until the computed and measured ligament lengths become comparable. We measure the ligament length from published scanning electron micrographs, when these data are not reported in literature. The remaining transport parameters are then directly determined from the local geometry model and accurately computed by numerically solving the appropriate boundary value problems.

First, we test the model against a series of measurements on polyurethane foam samples recently reported by Kino et $\mathrm{al}^{8}{ }^{8}$ Data (non-acoustical) for porosity $\phi$ and permeability $k_{0}$ were provided for three polyurethane foam samples, namely, 1, 2, and 5 (Table 6 of Ref. 8); together with a scanning electron micrograph per foam sample taken at magnification $\times 50$ (Fig. 1 of Ref. 8)-and from which rough estimates of the corresponding ligament lengths lying in the plane of observation might be obtained (to limit the 2D projection bias), yielding $L_{m} \pm \Delta L_{m}$. We next tested the quantitative accuracy of our model's predictions against those of semi-empirical models derived for fully and non-fully reticulated polyurethane foam samples. ${ }^{9}$ In this paper, Doutres, Atalla, and Dong (DAD) carried out extensive measurements. They provide expressions allowing correlations between macroscopic parameters and local geometry features of polyurethane foam samples from fifteen different materials, together with the normal incidence sound absorption coefficient for three of them (namely, M7, M9, and M11). Finally, data on various polyurethane foam samples, namely, $\mathrm{R}_{1}, \mathrm{R}_{2}$, and $\mathrm{R}_{3}$, were taken from Tables I and II of Ref. 14. We can, therefore, compare our model with three new complete sets of experimental data available in the literature (Refs. 8, 9, and 14).

The details on the numerical homogenization method used in this study were described elsewhere. ${ }^{14}$ Briefly, quasistatic viscous permeability measurements on real porous materials should respect the essentially laminar airflow entering and leaving the test specimen. The finite-element method provides approximate solutions to partial differential equations reflecting the polymeric foam's local geometry, assumed to be motionless. The scale separation allows the elimination of the coupling terms between visco-inertial and thermal dissipations. Locally, the fluid flow is described by incompressibility, and the fluid pressure is constant throughout a pore cross-section transverse to the compression wave propagation direction. The asymptotic transport coefficients were computed numerically from field averaging by solving the Stokes (static viscous permeability $k_{0}$, static viscous tortuosity $\alpha_{0}$ ), potential-flow (tortuosity $\alpha_{\infty}$, and viscous length $\Lambda$ ), and Laplace equations (static thermal permeability $k_{0}{ }^{\prime}$ also known as the inverse of the trapping constant $\Gamma$, static thermal tortuosity $\left.\alpha_{0}^{\prime}\right)$. Open porosity $\phi$ and thermal characteristic length $\Lambda^{\prime}$ also known as the generalized hydraulic radius are purely geometrical macroscopic parameters, respectively, derived from the fluid volume to bulk volume ratio and from twice the fluid volume to wetted surface area ratio. The linear response functions of the visco-thermal fluid entailed in a rigid porous medium and subjected to an infinitesimal oscillatory pressure gradient across the sample were estimated analytically using semi-phenomenological models, which are valid as long as the wavelength of sound (in the fluid) is much larger than the characteristic sizes of pores. In what follows, we test the results of the numerical model directly with the literature data.

\section{RESULTS}

Our method was tested against information available for a series of nine polyurethane foam samples that can be studied in two independent ways: by direct measurements of ligament lengths and thicknesses when the solid foam is free of solid film (Fig. 9(a) of Ref. 14), and using the iterative approach (Ref. 15) when the foam samples are not fully reticulated. These determinations yield the identification of the local characteristic lengths governing sound wave properties (Fig. 1). Fig. 1 also shows the localization of these characteristic lengths on the scanning electron micrographs. Our data show good agreement with the real foam sample micrographs. Fixing from measurements $\phi, k_{0}$, and $L_{m}$, or $L_{m}$ and the ligaments thickness $2 r$, our method ${ }^{14,15}$ yields a prediction for $\Lambda^{\prime}, \alpha_{0}, \Lambda, \alpha_{\infty}, k_{0}{ }^{\prime}, \alpha_{0}{ }^{\prime}$, in acceptable agreement with Refs. 8 and 9 (Tables I and II), but also in agreement with the microstructure. Note that there are only three samples chosen from DADs' fifteen because the measured values of acoustic properties are given by DAD for only three samples. In other words, the acoustical prediction's usefulness as a direct prediction of $\Lambda^{\prime}, \alpha_{0}, \Lambda, \alpha_{\infty}, k_{0}{ }^{\prime}, \alpha_{0}{ }^{\prime}$, based on two or three experimental measurements $\left(\phi, k_{0}\right.$, and $L_{m}$; or $L_{m}$ and $2 r$ ) might be fully established only for samples M7, M9, and M11 with Ref. 9.

From the macroscopic parameters, we obtain all the other acoustic quantities of the rigid porous material (Ref. 14, Appendix B]. Fig. 2 shows the sound absorption coefficient at normal incidence $\left(\mathrm{SAC}_{\mathrm{NI}}\right)$ as a function of frequency. At low frequencies, the $\mathrm{SAC}_{\mathrm{NI}}$ is governed by the fixed permeability $k_{0}$. At high frequencies, the $\mathrm{SAC}_{\mathrm{NI}}$ is dominated by the asymptotic high frequency parameters $(\Lambda$, $\alpha_{\infty}$, and $\left.\Lambda^{\prime}\right)$. In between, the effect of the elastic properties of the real foam samples may be observed. For instance, a dramatic rise of the $\mathrm{SAC}_{\mathrm{NI}}$ is observed for sample 1 around $1500 \mathrm{~Hz}$ (Fig. 2, top left). Such a feature in the experimental values of the $\mathrm{SAC}_{\mathrm{NI}}$ is the characteristic of local resonances of an elastic solid phase, which may be taken into account, for example, using classical Biot theory. ${ }^{16}$ 

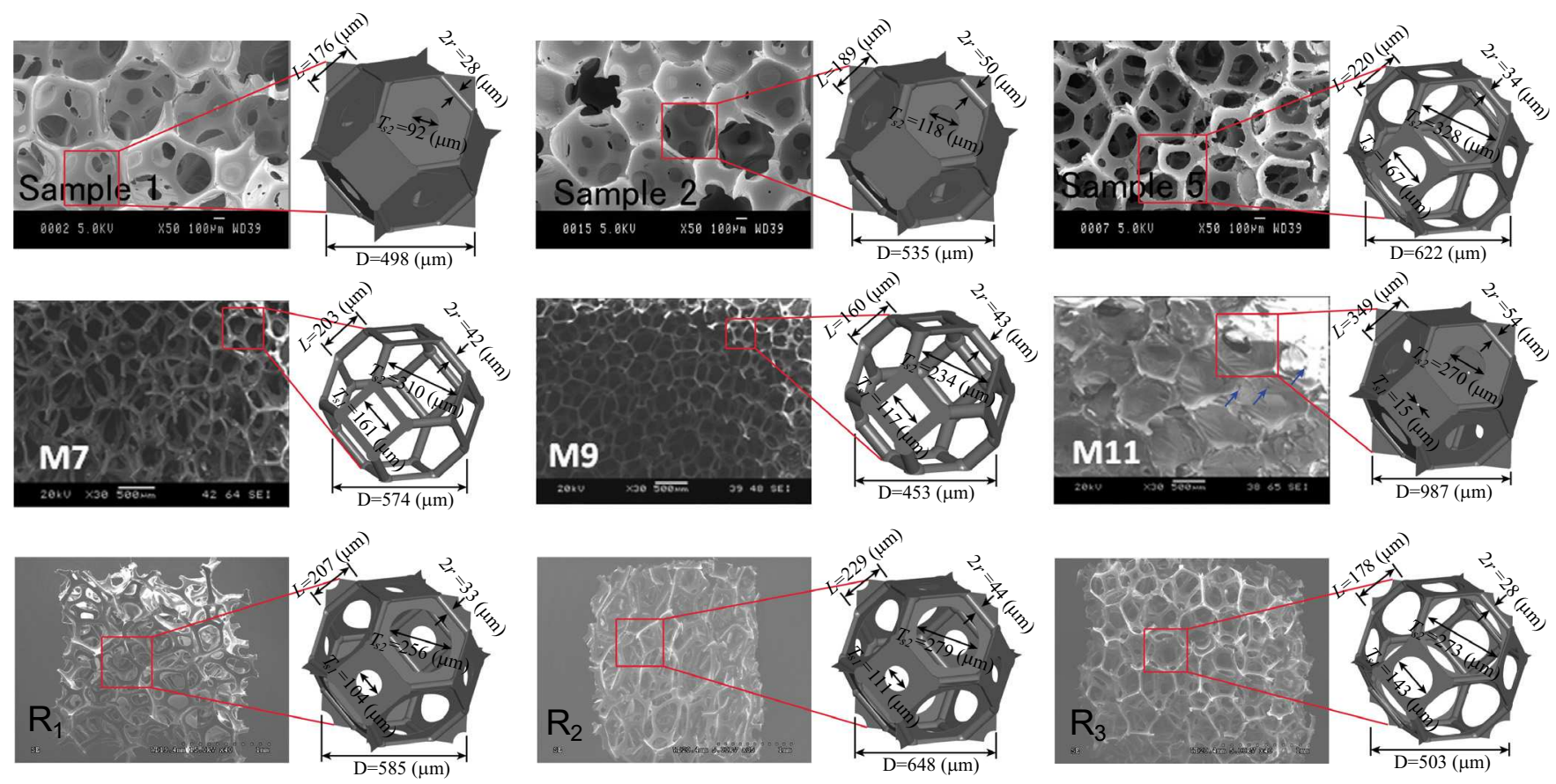

FIG. 1. SEM images and localization of specific unit cells to the critical paths (of the fluid flows) in real foam samples. (Top line) Micrographs and unit cells of sample 1, sample 2, and sample 5 in Ref. 8. (Middle line) Micrographs and unit cells of foam samples M7, M9, and M11 in Ref. 9. Arrows represent some positions of $T s_{1}$. The difficulty to visualize $T s_{1}$ in M11 is attributed to the low magnification of the corresponding SEM. (Bottom line) Micrographs and unit cells of foam samples $R_{1}, R_{2}$, and $R_{3}$ in Refs. 14 and 15 .

We shall now examine some elements based on experimental characterizations of the porous materials that can serve as a basis to simply interpret discrepancies between the experimental and numerical results, as shown in Fig. 2(a) (left and center). Such attempts can be found in the literature on the acoustics of porous materials where geometrical, transport, and elastic properties were related empirically to an elastoacoustic coupling criterion, the Frame Acoustical Excitability (FAE) ${ }^{17}$ For instance, because the permeability of sample 5 is very low (Table I) and its skeleton deformable (relatively low Young's modulus, Table I of Ref. 8), the elasto-acoustic coupling with the acoustic wave is strong, Fig. 2(a) (left). This corresponds to a high FAE, FAE $>2(\mathrm{~W} / \mathrm{kg})$. Although the Zwikker and Kosten decoupling frequency $f_{d}{ }^{18}$ indicates, in the frequency range of interest, the existence of partial decoupling between the solid and fluid phases of the porous sample, the FAE criterion also formulates this acoustical energy transfer to the solid phase in terms of the resonance frequency of the frame-borne wave. These are combined with a geometrical shape factor of the foam sample, in order to deal with the effect of the edge constraint and mounting conditions in a standing wave tube. Interestingly, because of the very low permeability of sample 5, a small penetrating depth of the wave is expected. This favors structural dissipation mechanisms by resonance of the frame corresponding to a high sound absorption peak, in addition to a surface absorption phenomenon. And it also explains why, contrary to less resistive foams for which a local sound absorption decrease is generally observed around the first quarter wavelength resonance frequency of the frame-borne wave, an absorption peak is observed in the same way as for closedcell elastic foams (Fig. 7 of Ref. 19).

In models of rigid-framed porous media, such jumps had been inferred by making empirical corrections to the Johnson-Champoux-Allard model to fit the experimental data that implied a resonance. ${ }^{8}$ Arguably this approach does better than the numerical method advocated in the current paper-at least for the samples 1 and 2. It should also be mentioned that sample 5 was prepared especially to remove all membranes. Moreover, Ref. 8 goes on to explain and elaborate on the presence of the "frame resonance in impedance tube data by reference to poroelastic theory. It is important to note, however, that Ref. 8 does not give a prediction for the acoustic properties of a porous material because it

TABLE I. Comparison between the experimental (Ref. 8) and numerical estimates based on three-dimensional unit-cells (Ref. 15).

\begin{tabular}{lccccccccccc}
\hline \hline Sample & Method & $\phi(-)$ & $\Lambda^{\prime}(\mu \mathrm{m})$ & $k_{0}\left(\times 10^{-9} \mathrm{~m}^{2}\right)$ & $\Lambda(\mu \mathrm{m})$ & $\alpha_{\infty}(-)$ & $k_{0}{ }^{\prime}\left(\times 10^{-9} \mathrm{~m}^{2}\right)$ & $L(\mu \mathrm{m})$ & $2 r(\mu \mathrm{m})$ & $\delta / \delta_{\max }(-)$ \\
\hline $1_{(3)}$ & Experimental estimates (Ref. 8) & 0.978 & 47 & 0.124 & 23 & 1.111 & - & $178 \pm 45$ & - \\
& Numerical computations & 0.98 & $156 \pm 42$ & 0.124 & $39 \pm 4$ & $2.856 \pm 0.551$ & $3.74 \pm 2.14$ & $176 \pm 52$ & $28 \pm 8$ & $1.25 \pm 0.13$ \\
$2_{(3)}$ & Experimental estimates (Ref. 8) & 0.946 & 70 & 0.242 & 35.1 & 1.1592 & - & $183 \pm 33$ & - & - & - \\
& Numerical computations & 0.945 & $168 \pm 24$ & 0.242 & $53 \pm 4$ & $2.333 \pm 0.246$ & $4.23 \pm 1.18$ & $189 \pm 31$ & $50 \pm 8$ & $1.15 \pm 0.10$ \\
$5_{(3)}$ & Experimental estimates (Ref. 8) & 0.978 & 324 & 5.81 & 161 & 1.0584 & - & $219 \pm 57$ & - & - \\
& Numerical computations & 0.98 & $482 \pm 175$ & 5.81 & $277 \pm 111$ & $1.050 \pm 0.053$ & $13.38 \pm 2.84$ & $220 \pm 46$ & $34 \pm 8$ & $0.1 \pm 0.15$ \\
\hline \hline
\end{tabular}


TABLE II. Comparison between semi-empirical (Ref. 9) and numerical estimates of multi-scale parameters derived from membrane-free ${ }^{14}$ (M7 and M9, direct microstructural data) and membrane-based ${ }^{15}$ (M11, iterative approach) three-dimensional unit-cells. (a) direct measurements and characterization techniques and (b) semi-empirical estimates.

\begin{tabular}{|c|c|c|c|c|c|c|c|c|c|c|c|}
\hline Sample & Method & & $\phi(-)$ & $\Lambda^{\prime}(\mu \mathrm{m})$ & $k_{0}\left(\times 10^{-9} \mathrm{~m}^{2}\right)$ & $\Lambda(\mu \mathrm{m})$ & $\alpha_{\infty}(-)$ & $k_{0}^{\prime}\left(\times 10^{-9} \mathrm{~m}^{2}\right)$ & $L\left(\mu_{\mathrm{m})}\right)$ & $2 r(\mu \mathrm{m})$ & $\delta / \delta_{\max }(-)$ \\
\hline \multirow[t]{6}{*}{ M7 } & \multirow[t]{4}{*}{ Semi-empirical estimates (Ref. 9) } & \multirow[t]{2}{*}{ (a) } & 0.987 & 435 & 10.97 & 269 & 1.042 & \multirow[t]{2}{*}{$\mathrm{Na}$} & 203 & 42 & 0 \\
\hline & & & \pm 0.01 & \pm 38 & \pm 0.79 & \pm 6 & \pm 0.006 & & \pm 43 & \pm 3 & \pm 0 \\
\hline & & \multirow[t]{2}{*}{ (b) } & 0.990 & 499 & 10.09 & 322 & 1.05 & \multirow[t]{2}{*}{$\mathrm{Na}$} & \multirow[t]{2}{*}{$\mathrm{Na}$} & \multirow[t]{2}{*}{$\mathrm{Na}$} & 0 \\
\hline & & & \pm 0.004 & \pm 216 & \pm 8.67 & \pm 139 & \pm 0 & & & & \pm 0 \\
\hline & \multirow{2}{*}{\multicolumn{2}{|c|}{ Numerical computations }} & 0.97 & 641 & 5.84 & 366 & 1.026 & 11.53 & 203 & 42 & 0 \\
\hline & & & \pm 0.02 & \pm 314 & \pm 3.99 & \pm 181 & \pm 0.015 & \pm 7.51 & \pm 43 & \pm 3 & \pm 0 \\
\hline \multirow[t]{6}{*}{ M9 } & \multirow[t]{4}{*}{ Semi-empirical estimates (Ref. 9) } & \multirow[t]{2}{*}{ (a) } & 0.968 & 268 & 4.94 & 183 & 1.059 & \multirow[t]{2}{*}{$\mathrm{Na}$} & 160 & 43 & 0 \\
\hline & & & \pm 0.01 & \pm 16 & \pm 0.16 & \pm 1 & \pm 0.001 & & \pm 20 & \pm 3 & \pm 0 \\
\hline & & \multirow[t]{2}{*}{ (b) } & 0.984 & 304 & 3.79 & 196 & 1.05 & \multirow[t]{2}{*}{$\mathrm{Na}$} & \multirow[t]{2}{*}{$\mathrm{Na}$} & \multirow[t]{2}{*}{$\mathrm{Na}$} & 0 \\
\hline & & & \pm 0.005 & \pm 80 & \pm 1.96 & \pm 51 & \pm 0 & & & & \pm 0 \\
\hline & \multirow[t]{2}{*}{ Numerical computations } & & 0.94 & 398 & 2.94 & 228 & 1.043 & \multirow{2}{*}{$\begin{array}{c}6.02 \\
\pm 2.47\end{array}$} & 160 & 43 & 0 \\
\hline & & & \pm 0.02 & \pm 119 & \pm 1.29 & \pm 68 & \pm 0.02 & & \pm 20 & \pm 3 & \pm 0 \\
\hline \multirow[t]{6}{*}{ M11 } & \multirow[t]{4}{*}{ Semi-empirical estimates (Ref. 9) } & (a) & 0.977 & 286 & 1.62 & 59 & 2.301 & \multirow[t]{2}{*}{$\mathrm{Na}$} & 358 & 85 & \multirow[t]{2}{*}{$\mathrm{Na}$} \\
\hline & & & \pm 0.01 & \pm 30 & \pm 0.16 & \pm 12 & \pm 0.092 & & \pm 10 & \pm 8 & \\
\hline & & (b) & 0.987 & 321 & 2.14 & 48 & 2.396 & $\mathrm{Na}$ & $\mathrm{Na}$ & $\mathrm{Na}$ & $\mathrm{Na}$ \\
\hline & & & \pm 0.003 & \pm 39 & \pm 1.29 & \pm 17 & \pm 0.455 & & & & \\
\hline & \multirow[t]{2}{*}{ Numerical computations } & & \multirow[t]{2}{*}{0.98} & 336 & \multirow[t]{2}{*}{1.62} & 96 & 1.907 & 16.30 & 349 & 54 & 0.95 \\
\hline & & & & \pm 8 & & \pm 7 & \pm 0.118 & \pm 0.92 & \pm 12 & \pm 16 & \pm 0.04 \\
\hline
\end{tabular}

uses semi-empirical fitted factors $\mathrm{N}_{1}$ and $\mathrm{N}_{2}$. The main objection to them in respect of the author's" work might be that they are applicable only to the tested materials and therefore not as potentially general as the parameters in the proposed approach. A second important consideration is that these factors cannot be simply interpreted, since they are not directly related to the pore structure.

In this work, we do not expect to predict the elastic behavior very close to the structural resonance, because the skeleton is assumed to be undeformable (this will be the topic of a forthcoming paper). The results (Fig. 2) show that most of the sound absorbing behaviors are well captured, within the error bars. It might be worth mentioning that the good agreement for foam sample M11 between the DAD semi-empirical estimates, and the standing wave tube measurements, is partly due to the fact that the data used to generate the model from fits are also the one taken to evaluate it. Another important remark is that DADs start from windows that are either opened or closed (Figs. 1 and 2 of Ref. 9), whereas our geometrical model relies on membrane sizes $\delta$ that are identical for all the windows (Fig. 1). One may, respectively, speak about a "binary" situation, as opposed to a rather "continuous" case (both of these modeling choices being induced by cellular morphology observations, and fabrication processes). A comparison between Fig. 2(b) (right) and Fig. 1(M11) suggests that an improvement of the sound absorption prediction is expected for a local geometry model that would combine the "binary" and "continuous" features in a common periodic unit cell. One can find many other porous materials in literature on the subject where such a cellular morphology could be applicable. Dupont et al. ${ }^{20}$ refer to air saturated materials containing dead-end porosities. The sound wave properties' prediction based on such a candidate needs more development and validation before its ultimate usefulness is made clear, and thus its discussion is beyond the scope of this paper.
Considering the small amount of input information $\{[\phi$, $\left.k_{0}, L_{m}\right]$ (Ref. 15), or [ $L_{m}$ and $\left.2 r\right]$ (Ref. 14, Fig. 9(a)) $\}$, the predictions are surprisingly close to the measured values of $\mathrm{SAC}_{\mathrm{NI}}$. This behavior is consistent with our macroscopic parameters (see Tables I and II, and Table I of Ref. 15), but the elastic resonance is not resolved. Unlike the previous experimental or semi-empirical determinations of macroscopic parameters for real foam samples, ${ }^{8,9}$ we essentially determine high frequency transport parameters directly from the unit cells, finding a local geometry model in agreement with the key multi-scale experimental data $\phi, k_{0}$, and $L_{m}$.

After an analysis of the proposed approach at the microstructural level, transport coefficients and acoustic properties were shown to be predicted, with good agreement with the experiment. The next question that must be addressed is: even if identifying local characteristic lengths is a good base of prediction for some transport coefficients and acoustic properties, does it work for analyzing transport coefficients in terms of microstructural parameters? In particular, does it work for polyurethane foam samples containing solid films or membranes which may have holes? From the computation of the viscous characteristic length $\Lambda$ (see Ref. 1), we can obtain a reduced geometrical representation $\Lambda / T_{s m}$ as a function of the closure rate of membranes $\delta / \delta_{\max }$ (Fig. 3(a)); the closure rate of membranes $\delta / \delta_{\max }$ being defined as the ratio between the actual membrane size and the maximum membrane size of the squared windows. First, let $\delta$ be the membrane size; and $\delta_{\max }$ the maximum membrane size such that the squared windows are entirely closed, $\delta_{\max }=L / 2-r$ (Fig. 1). One can see that the closure rate of membranes $\delta / \delta_{\max }$ may progress from an opened-cell structure without any solid film $(\delta=0)$ to a fully closed-cell structure when $\delta / \delta_{\max } \rightarrow 2.1$. More precisely, the maximum value of $\delta / \delta_{\max }$ depends on the porosity $\phi$. In the interval of $\phi$ from 0.9 to 0.99 , this maximum closure rate of membranes 

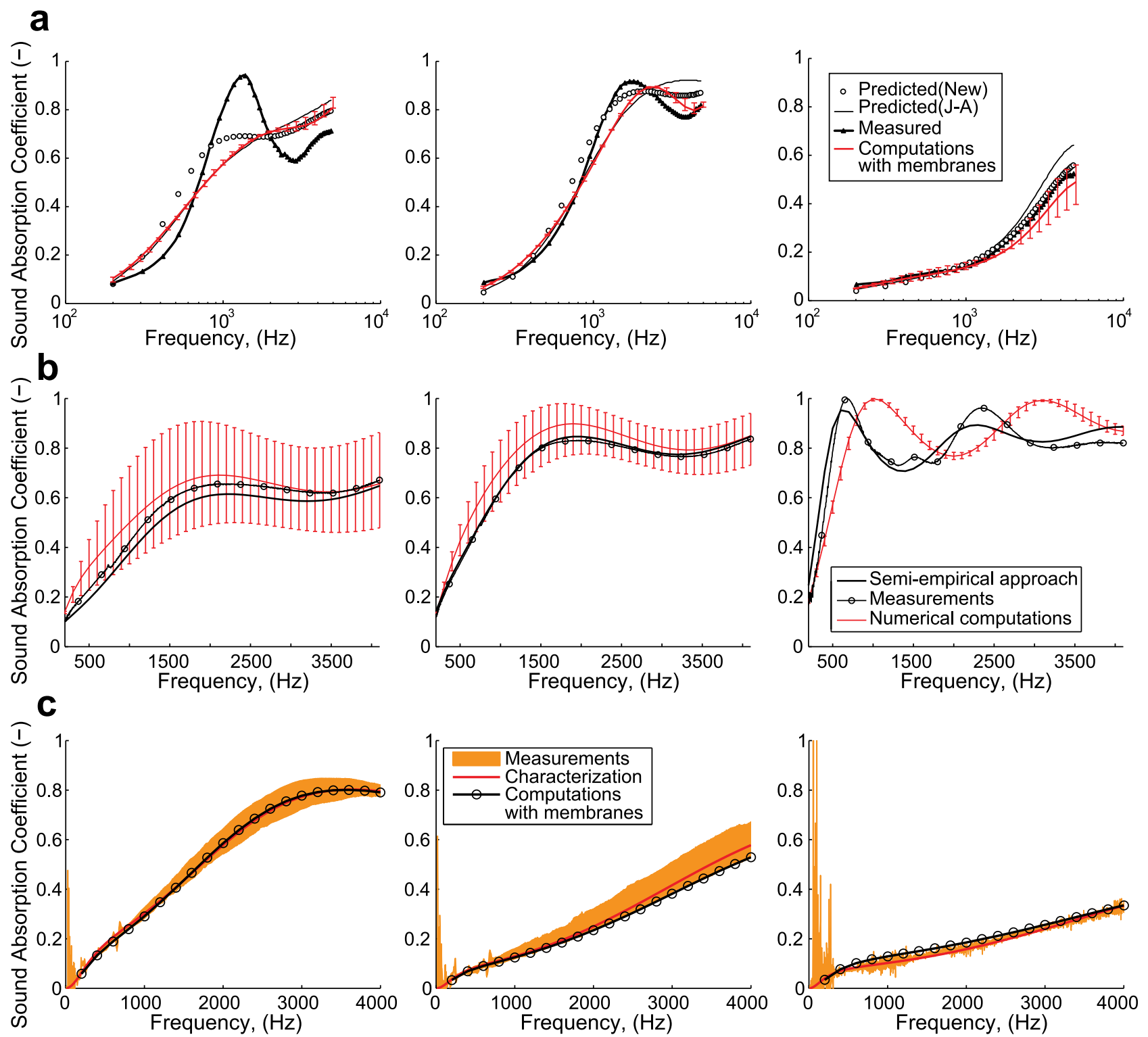

FIG. 2. Sound absorption coefficients at normal incidence $\left(\mathrm{SAC}_{\mathrm{NI}}\right.$ ) of real foam samples. (a) $\mathrm{SAC}_{\mathrm{NI}} \mathrm{vs}$ frequency from sample 1 (left), sample 2 (middle), and sample 5 (right) in Ref. 8. The red thin lines in (a) correspond to our computations when using the unit cells shown in Fig. 1 to be compared with standing wave tube measurements (black thick lines with triangles). Also shown in black thin lines and circles are, respectively, the so-called predicted (J-A) and predicted (New) $\mathrm{SAC}_{\mathrm{NI}}$ as obtained by Kino et al. in Ref. 8. The error bars in red are dominated by the statistical errors in the ligament lengths measurements, which were determined from SD uncertainties using micrographs shown in Fig. 1 (top line). These uncertainties, in turn, determine the corresponding uncertainties in the $\mathrm{SAC}_{\mathrm{NI}}$. (b) $\mathrm{SAC}_{\mathrm{NI}}$ vs frequency for foam samples M7 (left), M9 (middle), and M11 (right) in Ref. 9. Error bars: the uncertainty region determined from all possible combinations of input parameters (doublets or triplets of input parameters, where an input parameter can take three possible values: mean, mean $+\mathrm{SD}$, and mean $-\mathrm{SD}$ ). (c) $\mathrm{SAC}_{\mathrm{NI}}$ vs frequency for foam samples $\mathrm{R}_{1}$ (left), $\mathrm{R}_{2}$ (middle) and $\mathrm{R}_{3}$ (right) in Ref. 15.

$\Omega=20 \frac{\sqrt{3}(L / r)-2}{(L / r)-2}$ varies from 2.1 to 1.8 . Then, let $T_{s m}$ be the mean throat size, $T_{s m}=\left(T_{s l}+T_{s 2}\right) / 2$. In the interval of $\delta /$ $\delta_{\max }$ from 0 to 1 , two throat sizes exist, $T_{s 1}$ and $T_{s 2}$, corresponding to the small (squared) and large (hexagonal) windows. Thus, for $\delta / \delta_{\max }>1, T_{s 1}=0$ and only $T_{s 2}$ differs from zero. In the interval of $\delta / \delta_{\max }$ from 0 to 1 , the reduced representation of $\Lambda$ attains a maximum of $\Lambda / T_{s m}=1.4$ at $\Lambda / T_{s m}$ as $\delta / \delta_{\max }=1 / 20$, and then decreases at larger closure rates, as expected for a perfect fluid flow in a porous medium whose viscous length is dominated by the smallest interconnections. Around $\delta / \delta_{\max }=1$, a singular behavior is observed, corresponding to the closing of squared windows. In the interval of $\delta / \delta_{\max }$ from 1 to around 1.75 , the reduced representation of $\Lambda$ is close to unity, $\Lambda / T_{s m} \approx 1$, and therefore the viscous characteristic length is close to the radius of the opening between interconnected pores, $\Lambda \approx T_{s 2} / 2$. This is a key result, relating explicitly the viscous characteristic lengths $\Lambda$ to the throat size radius $T_{s 2} / 2$.

From the unit cell geometry and perfect fluid flow, we can obtain the thermal over viscous characteristic lengths ratio $\Lambda^{\prime} / \Lambda$ as a function of $\delta / \delta_{\max }$ (Fig. 3(b)). At low closure rates, $\Lambda^{\prime} / \Lambda$ is close to the typical macroscopic characteristic lengths ratio of a fibrous medium. Above $\delta / \delta_{\max }=1, \Lambda^{\prime} / \Lambda$ is always large compared with $\Lambda^{\prime} / \Lambda=2$. This shows that partially closed cell solid foams cannot be described in terms of a fibrous medium picture, although some sound absorbing properties agree surprisingly well with the expectation of a fibrous medium. ${ }^{8}$ Above $\delta / \delta_{\max }=1.25, \Lambda^{\prime} / \Lambda$ begins to 


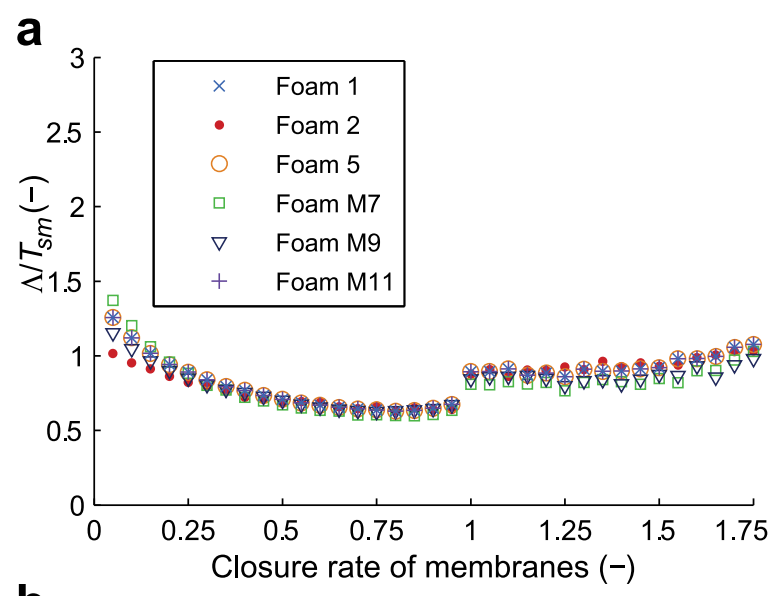

b

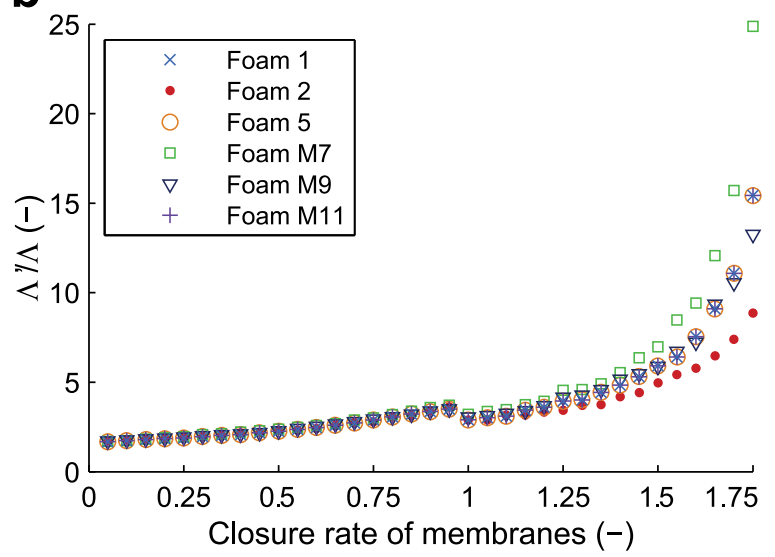

FIG. 3. $\Lambda / T_{s m}$ (a) and $\Lambda^{\prime} / \Lambda$ (b) vs closure rate of membranes for simulated foam samples of Refs. 8 and 9 shown in Fig. 1.

strongly increase over the value expected for a reticulated solid foam, which we interpret as an increasing contrast between the pore and interconnected throat sizes. Membranes have a strong overall contribution to this contrast. Although the closure rate of membranes and the closed pore content are not directly comparable, a similar trend is observable on DAD measurements which related the thermal over viscous lengths ratio to the closed pore content by a power law [see Fig. 6(f) and Eq. (7) of Ref. 9]. Above this $\delta / \delta_{\max }=1.25$, we also obtain a dependency of $\Lambda^{\prime} / \Lambda$ as a function of $\phi$.

\section{DISCUSSION AND CONCLUSION}

We performed numerical homogenization experiments of the compression wave propagation across an air-filled saturated rigid foam sample at the long wavelength regime, without any fits at micro- or macro-scales, enabling validation of the method for polyurethane foam samples. Similar unbiased methods can be applied to other porous materials, for example, aluminum foams with spherical cells ${ }^{21}$ or stratified foams made of recycled materials ${ }^{22}$ and porous bones.

The results of this comparative investigation are a milestone in the quantitative analysis and understanding of this problem. The computation of transport parameters-how sound propagation and dissipation properties relate to the cellular morphology-relies on determining the local velocity and temperature fields as a function of the low and high frequency asymptotic behaviors from idealized periodic unit cells. Assuming long wavelengths incident sound waves, we determined the local characteristic lengths, transport, and acoustic properties of solid foams with unprecedented multiscale consistency. In particular, we determined the high frequency transport parameters of the foam by relating the permeability and closure rate of membranes up to the measured cell size. This new method for characterizing solid foams improved the identification of local characteristic lengths governing sound wave properties compared to previous experiments, 8,9 and also allowed the observation of the idealized unit cell in a direct manner (see the Figs. 1 and 3). This work provides precise values of interdependent macroscopic parameters, such as $\phi, k_{0}, k_{0}{ }^{\prime}, \Lambda, \Lambda^{\prime}, \alpha_{0}, \alpha_{0}{ }^{\prime}$, and $\alpha_{\infty}$; which are essentially the physical signatures of the low Reynolds numbers hydrodynamics, electric conduction, and diffusion-controlled reaction processes; and remain controversial from the experimental point of view. ${ }^{23}$ Indeed, the numerical results are now accurate enough to benchmark novel experimental methods for the characterization of porous media properties problem.

An import step in our evaluation of the acoustic properties is the reduction of the disordered porous material to an equivalent 3D PUC. We remark that the critical path ideas that we used to obtain the scaling of the equivalent 3D PUC is essential for a correct understanding of the problem, and that correct local characteristic sizes can be obtained for the overall transport properties governing acoustic propagation at the upper scale if this feature is taken into account. An incorrect evaluation of the local characteristic sizes is obtained, for example, if one ignores the local heterogeneity in the pore sizes, and neglects the strong cross-section changes induced by the presence of membranes. In this picture, the 3D PUC linking the active sites of the real porous material is representative of the long-wavelengths acoustic properties; and, thus, the microstructure of the material can be optimized for specific purposes by adjusting the local geometry parameters of the model owing to the progress in manufacturing methods. ${ }^{24}$ In addition, the computations shed new light on the interpretation of the viscous and thermal characteristic lengths in terms of either the throat size (Fig. 3(a)) or the cell size (Fig. 3(b)); the general features of the numerical results are substantiated by the experimental data of the literature. In this sense, 3D PUC identification can serve as a kind of bridge that allows solving multi-scale problems that are intractable otherwise.

\section{ACKNOWLEDGMENTS}

We thank the authors of Ref. 9 for kindly providing us with their data and M.-H. Alexandre for language editing assistance. The authors are pleased to thank an anonymous referee, as well as K. Attenborough and R. Panneton who acted as referee of Minh Tan Hoang's Ph.D. dissertation, ${ }^{25}$ for valuable comments and suggestions that helped them to considerably improve the manuscript. The ANRT and Faurecia Interior Systems Acoustic TechCenter are gratefully acknowledged via Grant 748/2009. 
${ }^{1}$ D. L. Johnson, J. Koplik, and R. Dashen, "Theory of dynamic permeability and tortuosity in fluid-saturated porous media," J. Fluid Mech. 176, 379 (1987).

${ }^{2}$ Y. Champoux and J. F. Allard, "Dynamic tortuosity and bulk modulus in air-saturated porous media," J. Appl. Phys. 70, 1975 (1991).

${ }^{3}$ S. R. Pride, F. D. Morgan, and A. F. Gangi, "Drag forces of porous media acoustics," Phys. Rev. B 47, 4964 (1993).

${ }^{4}$ D. Lafarge, P. Lemarinier, J. F. Allard, and V. Tarnow, "Dynamic compressibility of air in porous structures at audible frequencies," J. Acoust. Soc. Am. 102, 1995 (1997). See also D. Lafarge, The equivalent fluid model (Chapter 6, Part II) in Materials and Acoustics Handbook, edited by C. Potel and M. Bruneau (Wiley, Chichester, 2009), pp 167-201.

${ }^{5}$ J. L. Auriault, "Heterogeneous medium. Is an equivalent macroscopic description possible?,” Int. J. Eng. Sci. 29, 785 (1991).

${ }^{6} \mathrm{~J}$. L. Auriault, "Dynamic behavior of a porous medium saturated by a Newtonian fluid," Int. J. Eng. Sci. 18, 775 (1980); R. Burridge and J. B. Keller, "Poroelasticity equations derived from microstructure," J. Acoust. Soc. Am. 70, 1140 (1981).

${ }^{7} \mathrm{C}$. Boutin, "Rayleigh scattering of acoustic wave in rigid porous media," J. Acoust. Soc. Am. 122, 1888 (2007).

${ }^{8}$ N. Kino, G. Nakano, and Y. Suzuki, "Non-acoustical and acoustical properties of reticulated and partially reticulated polyurethane foams," Appl. Acoust. 73, 95 (2012)

${ }^{9}$ O. Doutres, N. Atalla, and K. Dong, "Effect on the microstructure closed pore content on the acoustic behavior of polyurethane foams," J. Appl. Phys. 110, 064901 (2011).

${ }^{10}$ S. Gasser, F. Paun, and Y. Brechet, "Absorptive properties of rigid porous media: Application to face centered cubic sphere packing," J. Acoust. Soc. Am. 117(4I), 2090-2099 (2005); C. Perrot, F. Chevillotte, and R. Panneton, "Bottom-up approach for microstructure optimization of sound absorbing materials," J. Acoust. Soc. Am. 124, 940 (2008); O. Umnova, D. Tsiklauri, and R. Venegas, "Effect of boundary slip on the acoustical properties of microfibrous materials," J. Acoust. Soc. Am. 126, 1850 (2009); C.-Y. Lee, M. J. Leamy, and J. H. Nadler, “Acoustic absorption calculation in irreducible porous media: A unified computational approach,” J. Acoust. Soc. Am. 126, 1862 (2009); C.-Y. Lee, M. J. Leamy, and J. H. Nadler, "Frequency band structure and absorption predictions for multi-periodic acoustic composites," J. Sound Vibrat. 329, 1809 (2010); J. A. Kulpe, C.-Y. Lee, and M. J. Leamy, "Computation of acoustic absorption in media composed of packed microtubes exhibiting surface irregularity,” J. Acoust. Soc. Am. 130, 826 (2011).

${ }^{11}$ R. Panneton and X. Olny, "Acoustical determination of the parameters governing viscous dissipation in porous media," J. Acoust. Soc. Am. 119, 2027 (2006); X. Olny and R. Panneton, "Acoustical determination of the parameters governing thermal dissipation in porous media," J. Acoust. Soc. Am. 123, 814 (2008).

${ }^{12}$ V. Ambegaokar, B. I. Halperin, and J. S. Langer, "Hopping conductivity in disordered systems," Phys. Rev. B 4, 2612 (1971).
${ }^{13}$ A. J. Katz and A. H. Thompson, "Quantitative prediction of permeability in porous rock," Phys. Rev. B 34, 8179 (1986); M. Y. Zhou and P. Sheng, "First-principles calculations of dynamic permeability in porous media," Phys. Rev. B 39, 12027 (1989).

${ }^{14}$ C. Perrot, F. Chevillotte, M. T. Hoang, G. Bonnet, F.-X. Bécot, L. Gautron, and A. Duval, "Microstructure, transport, and acoustic properties of open-cell foam samples: Experiments and three-dimensional numerical simulations," J. Appl. Phys. 111, 014911 (2012).

${ }^{15}$ M. T. Hoang and C. Perrot, "Solid films and transports in cellular foams," J. Appl. Phys. 112, 054911 (2012).

${ }^{16} \mathrm{M}$. A. Biot, "Theory of propagation of elastic waves in a fluid-saturated porous solid. I. Low frequency range," J. Acoust. Soc. Am. 28, 168 (1956); M. A. Biot, "Theory of propagation of elastic waves in a fluid-saturated porous solid. II. Higher frequency range,” J. Acoust. Soc. Am. 28, 179 (1956).

${ }^{17}$ D. Pilon, R. Panneton, and F. Sgard, "Behavioral criterion quantifying the edge-constrained effects on foams in the standing wave tube," J. Acoust. Soc. Am. 114, 1980 (2003).

${ }^{18}$ C. Zwikker and C. W. Kosten, Sound-Absorbing Materials (Elsevier, Amsterdam, 1949).

${ }^{19}$ J.-L. Wojtowicki and R. Panneton, "Improving the efficiency of sealing parts for hollow body network," in Noise \& Vibration Conference and Exhibition, SAE Technical Paper No. 2005-01-2279 (2005).

${ }^{20}$ T. Dupont, P. Leclaire, O. Sicot, X. L. Gong, and R. Panneton, "Acoustic properties of air-saturated porous materials containing dead-end porosity," J. Appl. Phys. 110, 094903 (2011)

${ }^{21}$ Y. Li, X. Wang, X. Wang, Y. Ren, F. Han, and C. Wen, "Sound absorption characteristics of aluminum foam with spherical cells," J. Appl. Phys. 110, 113525 (2011).

${ }^{22}$ S. Mahasaranon, K. V. Horoshenkov, A. Khan, and H. Benkreira, "The effect of continuous pore stratification on the acoustic absorption in open cell foams," J. Appl. Phys. 111, 084901 (2012).

${ }^{23}$ K. V. Horoshenkov, A. Khan, F.-X. Bécot, L. Jaouen, F. Sgard, A. Renault, N. Amirouche, F. Pompoli, N. Prodi, P. Bonfiglio, G. Pispola, F. Asdrubali, J. Hübelt, N. Atalla, C. K. Amédin, W. Lauriks, and L. Boeckx, "Reproducibility experiments on measuring acoustical properties of rigid-frame porous media (round-robin tests)," J. Acoust. Soc. Am. 122, 345 (2007).

${ }^{24}$ A. Testouri, L. R. Arriaga, C. Honorez, M. Ranft, J. Rodrigues, A. van der Net, A. Lecchi, A. Salonen, E. Rio, R.-M. Guillermic, D. Langevin, W. Drenckhan, "Generation of porous solids with well-controlled morphologies by combining foaming and flow chemistry on a Lab-on-a-Chip," Colloids Surf., A 413, 17 (2012).

${ }^{25}$ M. T. Hoang, "Modélisation et simulation multi-échelle et multi-physique du comportement acoustique de milieux poroélastiques : Application aux mousses de faible densité," Ph.D. thesis, Université Paris-Est, 2012; translation in English: "Multi-scale and multi-physics modelization and simulation of the acoustical behavior of poroelastic media: Application to low density foams." 